\title{
Essai d'une complémentation alimentaire au flushing et au steaming de brebis Ouled Diellal par la caroube
}

\author{
Mebirouk-Boudechiche, L. ${ }^{\circledR} ;$ Bouhedja, N. ${ }^{2}$; Boudechiche, L. ${ }^{1}$ et Miroud, K. ${ }^{1}$ \\ 'Laboratoire d'Épidémio-Surveillance, Santé, Productions et Reproduction, Expérimentation et Thérapie Cellulaire des Animaux Domestiques et \\ Sauvages. Université Chadli Bendjedid El Tarf. El Tarf. Algerie. \\ ${ }^{2}$ Département des Sciences Vétérinaires. Université Chadli Bendjedid El Tarf. El Tarf. Algerie.
}

\section{MOTS CLÉ SUPPLÉMENTAIRES}

\section{Fluhsing.}

Steaming.

Brebis.

Complémentation.

Performance reproductive.

Performance productive.

Caroube.

Orge.

État corporel.

\section{RÉSUMÉ}

La présente étude a pour but la substitution d'un concentré à base de caroube entière à un concentré à base d'orge. Elle a concerné cinquante brebis, réparties aléatoirement en deux lots: un lot témoin ayant reçu une complémentation à base d'une ration $\mathrm{R} 1$ constituée de $30 \%$ son de blé et de $70 \%$ d'orge en grains au flushing puis au steaming, et un lot expérimental qui a reçu un concentré R2 à base de $30 \%$ de son de blé et de $70 \%$ de caroube entière. L'impact de cette complémentation a été évalué par les notes d'état corporel (NEC) à la lutte et à la mise bas, les performances de reproduction ainsi que la croissance des agneaux.

Les NEC ont été significativement identiques entre les deux lots à la lutte $(3,33$ vs. 3,48$)$ et à la mise bas $(2,87$ vs. 2,94). De même, les vitesses de croissance ont été significativement similaires $(p>0,05)$. Ainsi, les compléments énergétiques (orge vs. caroube) peuvent se substituer.

\section{Test of supplementation Ouled Diellal ewes by carob at flushing and steaming}

\section{SUMMARY}

This study aims to substitute a concentrate containing whole carob food to the concentrate containing barley. It concerned fifty ewes, randomly divided into two groups : a control group that received supplementation based on a ration R1 containing wheat bran $(30 \%)$ and barley grains $(70 \%)$ in the flushing and steaming, while experimental group received at the same time a ration R2 containing wheat bran $(30 \%)$ and whole carob $(70 \%)$. The impact of this complementation was evaluated by body condition score at mating, before lambing, reproductive performance and lamb growth.

The body condition scores in breeding were significantly identical between the two groups (3.33 vs. 3.48 ) and at lambing (2.87 vs. 2.94). Similarly, growth rates of lambs were not significantly different $(p>0.05)$. Thus, the energy supplements (barley vs. carob) can be substituted.

\section{INFORMACIÓN}

Cronología del artículo.

Recibido/Received: 12.5.2015

Aceptado/Accepted: 28.10.2015

On-line: 10.12.2015

Correspondencia a los autores/Contact e-mail:

boudechiche.lamia@gmail.com

\section{INTRODUCTION}

Au cours de leur cycle annuel d'élevage, les brebis passent par des périodes clés qu'il convient de bien préparer sur le plan alimentaire car elles conditionnent directement les performances du troupeau. En effet, l'alimentation est, d'une façon générale, l'un des principaux facteurs conditionnant les productions animales.

La période avant la lutte et celle avant les mises basses exigent, généralement, une supplémentation alimentaire en vue d'améliorer l'état de chair des femelles, ce qui a pour but d'améliorer sensiblement leurs performances en augmentant le taux d'ovulation et en réduisant la mortalité embryonnaire précoce (Paquay, 2004), ce qui aura pour conséquence un meilleur taux d'agnelage. En outre, l'alimentation avant la mise bas a une action déterminante sur la brebis et ses productions en conditionnant le poids des agneaux à la naissance ainsi que la production laitière de la brebis, notamment la sécrétion de colostrum (Teyssier et al., 1995) qui exige le recours aussi au steaming.

Cette alimentation intensive consiste en général à fournir principalement un apport supplémentaire de céréales à raison de 300 à $400 \mathrm{~g}$ par animal surtout si les conditions avant ces périodes clés sont insuffisantes ou 
si l'alimentation est faible à ce moment (Paquay, 2004), il peut s'agir principalement de l'orge qui est la céréale utilisée par excellence par les éleveurs algériens. Or cette dernière, qui est en Algérie quasiment importée d'Europe ou d'Amérique, tout comme le blé sont des produits boursiers, cotés dans les grandes places financières qui ne cessent de connaître un trend boursier haussier, accentué par un taux de change défavorable du dinar, ce qui a pour conséquence de renchérir le produit final.

Le prix élevé de cette céréale doit interpeller donc les éleveurs à valoriser des ressources locales et économiques, capables de concurrencer l'orge.

La caroube, qui est un fruit d'origine méditerranéenne poussant à l'état sauvage, est employée depuis des temps antiques dans le pourtour méditerranéen où elle est aussi bien utilisée pour l'alimentation animale qu'humaine.

Elle suscite actuellement beaucoup d'intérêt à l'échelle internationale où elle est exploitée en industrie agro-alimentaire et en pharmacologie. Elle n'est pas non plus sans intérêt en Algérie qui se place au huitième rang des producteurs de caroube à l'échelle mondiale, avec une production de $3600 \mathrm{~T}$ (Food and Agriculture Organization Statistics [FAOSTAT], 2010). En effet, cet arbre d'une importance économique considérable, génère beaucoup de produits intéressants (pulpe, graines, gomme) qui font 1'objet de transactions commerciales importantes en direction de l'Europe.

Sur le plan scientifique, les rares travaux qui ont concerné l'utilisation de la caroube en alimentation animale se sont limités à l'incorporation de sa pulpe dans les rations pour engraissement (Guessous et al., 1988), ou de l'extrait de graine en cuniculture (Teillet et al., 2011), outre, des essais ont aussi été menés dans le domaine de l'industrie agroalimentaire (Avallone et al., 1997; Biner et al., 2007). Cependant, Il n'y a pas eu à notre connaissance, beaucoup de travaux sur l'utilisation de la caroube entière (pulpe et graine) en période de flushing ou de steaming des brebis. Les travaux les plus connus traitent de l'utilisation du concentré classique à base d'orge chez ces catégories d'animaux.

C'est dans un souci de réduction des coûts de l'alimentation animale, qui est de loin, le poste le plus important des dépenses en élevage, tout en maintenant de bonnes performances que s'inscrit en filigrane l'objet de cet essai qui vise à étudier l'influence du type de complément énergétique (caroube vs. orge) par substitution totale sur les performances zootechniques (état corporel, vitesse de croissance des agneaux) et reproductives (taux de gestation, de fertilité, de prolificité, de fécondité, et de productivité numérique) des brebis Ouled Djellal au flushing et au steaming.

\section{MATÉRIEL ET MÉTHODES}

\section{ANIMAUX ET DISPOSITIF EXPÉRIMENTAL}

L'étude a porté sur 50 brebis multipares vides, de race Ouled Djellal âgées de 3-4 ans.

La race étudiée est la plus importante race ovine en Algérie. Elle représente environ $63 \%$ du cheptel national (Boucif et al., 2007). Elle est adaptée au milieu steppique et elle présente des qualités exceptionnelles pour la production de viande (Abbas et al., 2002; Cabée, 1959; Chellig, 1992). Selon Dehimi et al. (2001), cette race ne présente pas d'anoestrus saisonnier et peut être fécondée tout au long de l'année.

Toutes les brebis sélectionnées pour l'étude n'avaient aucun antécédent pathologique majeur et présentaient une bonne activité de reproduction. Elles ont été traitées contre les parasitoses internes et externes et vaccinées contre les clostridioses animales et les principales maladies des petits ruminants conformément au programme national de vaccination. Le troupeau de brebis était homogène sur les critères suivants :

- Deux périodes principales de lutte: au printemps et en automne, avec monte naturelle;

- Deux agnelages par an sur deux périodes aoutseptembre et décembre-janvier;

- Conduite principale en système extensif, mais complémentation de certains animaux à haut besoin.

Les brebis ont été réparties aléatoirement en deux lots; un lot témoin et un autre expérimental et ont fait l'objet de deux essais successifs: un essai flushing et un autre steaming.

\section{L'ESSAI FLUSHING}

Toutes les brebis s'alimentaient de manière analogue à partir d'une prairie naturelle et des chaumes de céréales (orge).

D'un point de vue alimentaire, la différence entre les deux lots résidait dans le fait que, pendant la période du flushing d'une durée de 4 semaines, le lot témoin recevait quotidiennement en complément du pâturage une complémentation par une ration R1 constituée de: $30 \%$ de son de blé, $0 \%$ de caroube et $70 \%$ d'orge en grains, alors que le lot expérimental recevait une ration R2 constituée de $30 \%$ de son de blé, $70 \%$ de caroube et $0 \%$ d'orge. Les deux régimes n'ont différé que par la nature des compléments énergétiques apportés (caroube vs. orge).

Après la mise en lot, une période de transition de 15 jours a été ménagée pour les brebis, au cours de laquelle les proportions des régimes expérimentaux ont été progressivement augmentées pour atteindre $500 \mathrm{~g} /$ animal/jour. L'eau était disponible à volonté.

Des béliers entiers et de bonne libido ont été utilisés pour les montes; ils ont été maintenus séparés des femelles sans aucun contact physique, visuel ou olfactif avec les brebis afin de bénéficier de l'effet bélier et ont aussi été soumis au flushing en recevant quotidiennement un mois avant la lutte $400 \mathrm{~g}$ d'orge en grains.

A la mise à la lutte de printemps, un sexe ratio de $1 / 10$ a été maintenu durant quatre cycles oestriens.

Afin de s'assurer de l'état de gestation des femelles et estimer l'âge de celle-ci pour entreprendre le steaming, un diagnostic de gestation a été réalisé à 79 jours de gestation, avec un échographe portable WED 3000, 
muni d'une sonde linéaire abdominale d'une fréquence de 5,5 MHz.

\section{L'ESSAI STEAMING}

Toutes les femelles gestantes ont été retenues pour cet essai qui consistait à leur présenter un foin de vesce avoine pendant les deux derniers mois de gestation, coïncidant aux mois d'Aout-septembre, la différence d'un point de vue alimentaire résidait dans le fait que, les femelles du lot témoin recevaient en plus du fourrage une complémentation quotidienne par le concentré (R1) alors que les femelles du lot expérimental recevaient une complémentation par le concentré (R2) à raison de $500 \mathrm{~g} /$ brebis/jour. Les deux régimes dans cet essai n'ont différé que par la nature des compléments énergétiques apportés (caroube vs. orge).

La période de transition de 15 jours a aussi été respectée pour cet essai.

La caroube utilisée dans les deux essais était constituée de la gousse entière (pulpe et graines), elle a été grossièrement broyée et mélangée au son dans la ration du lot expérimental. Un échantillon représentatif de chaque ration (R1 et R2) a fait l'objet d'une analyse de sa composition chimique selon les méthodes AOAC (1990), il en est de même pour le pâturage fréquenté par les femelles durant le flushing. Ainsi, des analyses de la composition chimique ont porté sur la matière sèche (MS), les cendres brutes (MM), la matière azotée totale (MAT) et la cellulose brute (CB).

Pour prévoir les teneurs en unité fourragère viande (UFV) et unité fourragère lait (UFL) des rations (R1 et R2), nous avons utilisé les équations de Sauvant (1981) pour les concentrés. Tandis que pour prévoir les valeurs énergétiques (UFL et UFV), les valeurs de protéines digestibles intestinales (PDI) et la digestibilité de la matière organique $(\mathrm{dMO}) \mathrm{du}$ pâturage à partir de sa composition chimique, nous avons utilisé les équations de Baumont et al. (2007) et Demarquilly et al. (1981).

\section{ObSERVATIONS ET MESURES}

\section{NOTATION D'ÉTAT CORPOREL}

concernant les deux essais, toutes les femelles du troupeau ont été soumises à des notations périodiques d'état corporel. Ainsi, leur état corporel a été estimé à deux reprises pour chaque essai: Un mois avant la lutte $\left(\mathrm{NEC}_{\mathrm{f}} \mathrm{I}\right.$, note d'etat corporel initiale avant flushing) puis au moment de la lutte $\left(\mathrm{NEC}_{\mathrm{f}} \mathrm{F}\right.$, note d'etat corporel finale après flushing), soit avant et après la suralimentation.

Et deux mois avant la mise-bas (NECs I, note d'etat corporel initiale avant steaming) puis au moment des agnelages (NECs F, note d'etat corporel finale après steaming), soit avant et après la suralimentation.

Ces notations a été estimée par palpation de la région lombaire et caudale selon le protocole et la méthodologie établis au cours de la réunion Agrimed - CIHEAM FAO du groupe de travail sur l'état corporel des petits ruminants qui attribue des notes à 0,25 point près (Morand-Fehr et al., 1990).

\section{ÉVALUATION DES PARAMĖTRES DE REPRODUCTION}

Les performances de reproduction des brebis ont été évaluées à partir des taux de fertilité, de fécondité, de prolificité et de productivité numérique des deux lots d'animaux.

\section{ÉVALUATION DES POIDS DES AGNEAUX}

Pour étudier l'effet des compléments énergétiques distribués en fin de gestation sur la croissance des agneaux, les mesures de poids ont été limitées à une période où celle-ci dépend de la production laitière de la mère. Tous les agneaux ont été pesés à la mise bas et régulièrement à intervalles de dix jours, à savoir: à la naissance (J0), à 10 jours (J10), à 20 jours (J20), à 30 jours (J30), à 40 jours (J40) et à 50 jours (J50) de vie.

\section{ANALYSES STATISTIQUES}

L'analyse des résultats des performances de reproduction des brebis obtenues, exprimés en pourcentage $(\%)$, a été réalisée suivant le test de khi-carré $\left(\chi^{2}\right)$ avec ou sans la correction Fisher-Yates, basé sur le travail de Brown (1988). Les comparaisons entre les paramètres de reproduction des deux lots ont été faites par le test de Student Newman-Keuls.

Les résultats portant sur l'état corporel des femelles ont été soumis à une analyse de variance à un critère (rations) de classification du model linaire général uni varié (GLM, SPSS version 17.0, 2008) en utilisant les notes d'état corporels initiales (NEC I) comme facteur de covariance afin d'éliminer leur influence sur les notes d'état corporels finales (NEC F). Les résultats des performances zootechniques sont exprimés par la moyenne \pm 1 'écart-type de la moyenne ( $\mathrm{m} \pm \mathrm{ECTM})$.

Une analyse de la régression a permis de mettre en évidence la relation entre la note d'état corporel des femelles à la mise bas et le poids des agneaux à la naissance, à J10, J20, J30, J40 et J50, de même qu'entre les poids initiaux des agneaux et leurs poids de J20 à J50.

\section{RÉSULTATS ET DISCUSSION}

\section{COMPOSITION CHIMIQUE ET VALEURS NUTRITIVES DU PÂTURAGE} ET DES RATIONS INGÉRÉES

Les valeurs énergétiques et protéiques du pâturage de printemps sont de 0,67 UFL et de $67,53 \mathrm{~g} / \mathrm{kg}$ de MS de PDIE (tableau I), cependant, on remarquera la faible teneur en PDIA du fourrage $(23,68 \mathrm{~g} / \mathrm{kg}$ de MS, ce qui est imputable à sa faible teneur en protéines brutes $(8,13 \%$ MS) (tableau I).

Les deux rations alimentaires présentent une bonne valeur énergétique $(0,95$ et $0,90 \mathrm{UFL} / \mathrm{kg}$ MS) (tableau I), respectivement pour les rations à base d'orge et de caroube, avec une supériorité pour la première ration.

La composition chimique de la ration à base de caroube entière fait ressortir une meilleure teneur en protéines brutes qu'avec une ration à base de pulpe de caroube seule $(15,12$ vs. $11,16 \% \mathrm{MS})$, ceci est sans doute dû à la richesse des graines en protéines $(30,25 \%$ MS) (Gaouar, 2011). 
Tableau I. Caractéristiques nutritionnelles du pâturage et des concentrés utilisés dans l'essai (Nutritional characteristics of pasture and rations of the study).

\begin{tabular}{lcccc}
\hline & & R2 & R1 & Pâturage \\
\hline MS & $(\%)$ & 84,34 & 84,63 & 27,06 \\
\hline MM & & 4,46 & 3,66 & 12,7 \\
MAT & (\% de la MS) & 15,12 & 11,16 & 8,13 \\
MG & 2,5 & 3,2 & 2,00 \\
CB & & 7,4 & 6,05 & 35,0 \\
\hline UFL & & 0,90 & 0,95 & 0,67 \\
UFV & (par kg de MS) & 0,89 & 0,94 & 0,59 \\
\hline PDIN & & & & 53,87 \\
PDIE & & & & 67,53 \\
PDIA & (g/kg de MS) & & & 23,68 \\
UEM & & & & 1,21
\end{tabular}

$M S=$ Matière sèche; $M M=$ Matière minérale; $M A T=$ Matières azotées totales; $\mathrm{MG}=$ Matière grasse; $\mathrm{CB}=$ Cellulose brute; UFL= Unité fourragère lait; UFV= Unité fourragère viande; PDIN= Protéines digestibles intestinales permises par l'azote; PDIE= Protéines digestibles intestinales permises par l'énergie; $P D I A=$ Protéines digestibles intestinales d'origine alimentaire; UEM= Unité d'encombrement mouton; R1= concentré du lot témoin (orge et son); R2= concentré du lot expérimental (caroube et son).

Tableau II. Effet de la complémentation sur l'évolution des notes d'état corporel au flushing (Moyennes \pm ECTM) (Effect of yhe complementation on body condition sore evolution at flushing (Means \pm ECTM)).

\begin{tabular}{lrrcc}
\hline Paramètres & $\mathrm{LT} n=(25)$ & $\mathrm{LE} n=(25)$ & $\mathrm{p}$ & Signification \\
\hline $\mathrm{NEC}_{\mathrm{f}} \mathrm{I}$ & $2,73 \pm 0,395$ & $2,95 \pm 0,235$ & 0,912 & $\mathrm{NS}$ \\
$\mathrm{NEC}_{\mathrm{f}} \mathrm{F}$ & $3,33 \pm 0,294$ & $3,48 \pm 0,336$ & 0,555 & $\mathrm{NS}$ \\
$\mathrm{NEC}_{\mathrm{f}} \mathrm{F}-\mathrm{NEC}_{\mathrm{f}} \mathrm{I}$ & $+0,60 \pm 0,264$ & $+0,53 \pm 0,339$ & 0,397 & $\mathrm{NS}$ \\
\hline
\end{tabular}

$\mathrm{ECTM}=$ Écart type de la moyenne; LT= Lot témoin; LE= Lot expérimental; $\mathrm{NEC}_{\mathrm{f}} \mathrm{l}=$ note d'etat corporel initiale avant flushing; $\mathrm{NEC}_{\mathrm{f}}$ $\mathrm{F}=$ note d'etat corporel finale après flushing.

INFLUENCE DE LA NATURE DU COMPLÉMENT ÉNERGÉTIQUE SUR L'ÉTAT CORPOREL DES BREBIS AU FLUSHING

L'évolution des NEC (notes d'état corporel) entre le début et la fin du flushing n'a pas été significativement différente entre les deux lots $(\mathrm{p}=0,555)$, avec des gains en note d'état corporel $(+0,60$ vs $+0,53$ point) respectivement pour les lots recevant de l'orge et de la caroube dans leurs rations $(\mathrm{p}=0,397)$ (tableau II).

Le flushing réalisé s'est d'autant avéré efficace puisque les $\mathrm{NEC}_{\mathrm{f}} \mathrm{I}$ étaient comprises entre 2,5 et 3 selon les recommandations de Bocquier et al. (1988) et Dudouet (2003).
Les deux régimes alimentaires se sont répercutés positivement sur l'état corporel des femelles des deux lots, ce qui s'est caractérisé par une amélioration de leur état de chair à travers l'amélioration de leur note d'état corporel.

Étant donné que la NEC mesure l'importance du tissu adipeux sous-cutané (Nicoll, 1981), les changements d'état corporel reflètent donc les variations de l'état des réserves corporelles, l'intérêt de ces dernières est connu, notamment chez les femelles reproductrices. Dedieu et al. (1989) et Torre et al. (1991) ont noté une relation positive entre les réserves corporelles et les taux d'ovulation, de fertilité et de prolificité chez les ovins.

Dans notre étude, les compléments énergétiques (Orge vs. Caroube) ont contribué à améliorer l'état de chair des femelles des deux lots de façon similaire $(p=$ $0,555)$.

Les réserves corporelles permises par les deux régimes alimentaires témoignent d'un bon état nutritionnel des femelles.

Le maintien de l'état corporel est largement assuré pour les brebis ayant reçu une complémentation au pâturage aussi bien en caroube qu'en orge. Ceci s'explique sans doute par les bonnes valeurs énergétiques des deux compléments qui ont permis le maintien, voire une légère augmentation des réserves corporelles en énergie sous forme de lipides, les NEC étant corrélées avec la teneur en lipides de l'organisme entier (Morand-Fehr et al., 1990).

\section{EFFET DU RÉGIME ALIMENTAIRE SUR LES PERFORMANCES DE REPRODUCTION DES BREBIS}

Les paramètres de reproduction des brebis en fonction de la nature du complément énergétique sont présentés respectivement dans le tableau III.

Les résultats globaux de fécondité et de prolificité obtenus, bien qu'ils soient à l'avantage des femelles complémentées par la caroube, ont été cependant statistiquement identiques ( $p>0,05)$.

La nature du complément énergétique (caroube vs. orge) n'a pas eu d'effet significatif sur les paramètres de reproduction des brebis, néanmoins, nous avons pu obtenir dans cet essai des paramètres supérieurs au standard de la race, à savoir fertilité $88 \%$, fécondité $93 \%$ et prolificité $110 \%$ selon Dehimi (2001).

Cette situation est imputable à la distribution de suppléments énergétiques aux brebis avant la période de lutte qui a permis d'améliorer les taux d'ovulation, de fécondation et l'implantation embryonnaire et par conséquent les taux de fertilité, de prolificité et de fécondité.

Tableau III. Les performances de reproduction des deux lots de brebis en \% (Reproductive performances of two groups of sheep in \%).

\begin{tabular}{|c|c|c|c|c|c|c|}
\hline & Taux de gestation & Taux de fertilité & Taux de fécondité & Taux de prolificité & Taux de mortalité & Productivité numérique \\
\hline LT & $100^{\mathrm{a}}$ & $100^{a}$ & $100^{\mathrm{a}}$ & $100^{\mathrm{a}}$ & $13,33^{\mathrm{a}}$ & $87^{a}$ \\
\hline LE & $100^{a}$ & $100^{a}$ & $113^{a}$ & $113^{a}$ & $23^{a}$ & $87^{a}$ \\
\hline
\end{tabular}

$\mathrm{LT}=$ Lot témoin; $\mathrm{LE}=$ Lot expérimental; Les moyennes d'une même colonne portant des lettres identiques sont significativement similaires au seuil de $5 \%$. 
Tableau VI. Effet de la complémentation sur les paramètres de mesure au steaming (Moyennes \pm ECTM) (Effect of supplementation on the measurement parameters to steaming (Means $\pm E C T M)$ ).

\begin{tabular}{lrccc}
\hline Paramètres & $\mathrm{LT} n=(25)$ & LE $n=(25)$ & $\mathrm{p}$ & Signification \\
\hline $\mathrm{NEC}_{\mathrm{s}} \mathrm{I}$ & $2,84 \pm 0,411$ & $2,87 \pm 0,413$ & 0,910 & $\mathrm{NS}$ \\
$\mathrm{NEC}_{\mathrm{s}} \mathrm{F}$ & $2,96 \pm 0,364$ & $2,94 \pm 0,406$ & 0,904 & $\mathrm{NS}$ \\
$\mathrm{NEC}_{\mathrm{s}} \mathrm{F}-\mathrm{NEC}_{\mathrm{s}} \mathrm{I}$ & $+0,12 \pm 0,234$ & $+0,07 \pm 0,359$ & 0,758 & $\mathrm{NS}$
\end{tabular}

ECTM=Écart type de la moyenne; $\mathrm{LT}=$ Lot témoin; $\mathrm{LE}=$ Lot expérimental; $\mathrm{NEC}_{\mathrm{s}} \mathrm{I}=$ Note d'etat corporel initiale avant steaming; $\mathrm{NEC}_{\mathrm{s}} \mathrm{F}=$ Note d'etat corporel finale après steaming.

En outre, Le facteur état corporel est l'un des plus déterminants sur le taux de fertilité, en effet, les brebis des deux lots avaient, au moment de la lutte, un bon état d'embonpoint, avec des NEC de 3,33 et 3,48 respectivement pour les brebis du lot témoin et du lot expérimental, ce qui a influencé positivement les taux de fertilité et de prolificité.

Theriez (1984) a également trouvé que la fertilité, la prolificité dépendent fortement de l'état corporel des femelles à la lutte.

Ceci reflète bien l'effet notable exercé par les compléments énergétiques: caroube et orge sur ces paramètres et encourage au remplacement de l'orge par la caroube.

En comparant les résultats de productivité numérique obtenus pour ces troupeaux, ils semblent en dessous des seuils recommandés pour les races dessaisonnées, agnelant en automne-hiver (>125\%) (Dirand, 2007).

\section{INFLUENCE DU TYPE DE COMPLÉMENT ÉNERGÉTIQUE (CAROUBE VS. ORGE) AU STEAMING SUR L'ÉTAT DE CHAIR DES BREBIS AU STEAMING}

L'évolution des notes d'état corporel (NEC) entre la période de fin de gestation et la mise bas n'a pas été significativement $(p=0,904)$ différente entre les deux lots qui ont présenté un léger gain $(+0,12$ vs. $+0,07$ respectivement pour les lots témoin et expérimental) (tableau IV).

Les femelles en fin de gestation ont tendance à puiser sur leurs réserves corporelles, l'intérêt du steaming réside justement dans la reconstitution de ces réserves corporelles qui seront principalement localisées au niveau du tissu adipeux et mobilisées en fin de gestation pour la croissance fœtale et le début de la lactation alors que la capacité d'ingestion de la brebis est limitée (Teyssier 1995) étant donné que la NEC mesure l'importance du tissu adipeux sous-cutané (Nicoll 1981), ces changements d'état corporel reflètent donc les variations de l'état des réserves corporelles.

Le maintien de l'état corporel est largement assuré pour les brebis ayant reçu aussi bien la complémentation à base d'orge ou de caroube. Ceci s'explique sans doute par la valeur énergétique de ces deux compléments qui a permis le maintien, voire une légère augmentation des réserves corporelles en énergie sous forme de lipides.
Tableau V. Effet du type de complément énergétique (caroube vs. Orge) sur le poids des agneaux et leur croissance pondérale ( $m \pm$ écart type) (Effect of type of energy supplement (Carob vs. Barley) on lamb weights and weight gain $(\mathrm{m} \pm \mathrm{SD}))$.

\begin{tabular}{lccc}
\hline Paramètres $(\mathrm{kg})$ & $\begin{array}{c}\text { Lot témoin } \\
(\mathrm{n}=22)\end{array}$ & $\begin{array}{c}\text { Lot expérimental } \\
(\mathrm{n}=22)\end{array}$ & $\mathrm{p}$ \\
\hline Poids moyen à J0 & $3,79 \pm 0,203$ & $3,75 \pm 0,339$ & 0,697 \\
GMQ 0-10j & $251,50 \pm 52,78$ & $277,67 \pm 55,87$ & 0,378 \\
Poids moyen à J10 & $6,31 \pm 0,628$ & $6,53 \pm 1,179$ & 0,580 \\
GMQ 10-20j & $202,75 \pm 52,00$ & $186,50 \pm 57,94$ & 0,650 \\
Poids moyen à J20 & $8,34 \pm 1,124$ & $8,39 \pm 1,614$ & 0,925 \\
GMQ 20-30j & $161,92 \pm 52,35$ & $166,00 \pm 49,49$ & 0,861 \\
Poids moyen à J30 & $9,96 \pm 1,24$ & $10,05 \pm 2,02$ & 0,891 \\
GMQ 30-40j & $146,08 \pm 44,04$ & $177,92 \pm 48,79$ & 0,227 \\
Poids moyen à J40 & $11,42 \pm 1,492$ & $11,83 \pm 2,309$ & 0,608 \\
GMQ 40-50j & $208,00 \pm 48,03$ & $187,67 \pm 48,49$ & 0,601 \\
Poids moyen à J50 & $13,50 \pm 1,801$ & $13,71 \pm 2,853$ & 0,831 \\
\hline
\end{tabular}

Tout comme dans l'essai flushing, les deux compléments énergétiques ont eu le même effet positif sur l'amélioration de l'état de chair des femelles en fin de gestation.

\section{RELATIONS ENTRE LES NOTES D'ÉTAT CORPOREL ET LA CROISSANCE DES AGNEAUX}

Pour les brebis des deux lots, le poids des portées à la naissance est pratiquement le même $(3,79$ vs. 3,75 respectivement pour les lots témoin et expérimental) $(p=0,697)$ (tableau V).

Cependant, dans les conditions d'élevage étudiées, les notes d'état corporel des brebis Ouled Djellal ne sont pas liées de manière significative aux poids des agneaux à la naissance $\left(p=0,456, R^{2}=2,5 \%\right)$. Ce résultat est identique à celui trouvé par d'autres auteurs (Molina et al., 1991) qui n'ont pas enregistré d'effets significatifs de la note des brebis à la mise bas sur le poids des agneaux à la naissance mais pour d'autres races et dans des systèmes d'élevage différents. Ce qui mène à conclure que la complémentation aussi bien par l'orge que par la caroube des femelles en fin de gestation a amélioré l'état corporel des brebis Ouled Djellal complémentées, mais n'a pas eu d'effet significatif sur le poids des agneaux à leur naissance.

Il en est de même pour les poids vifs à J10, J20, J30, J40 et J50 qui n'ont pas été influencé par le type de complément énergétique puisqu'ils ont été significativement identiques aussi bien pour les agneaux du lot témoin qu'expérimental. L'alimentation durant cette phase est exclusivement à base de lait, et une production laitière identique des femelles pourrait être à l'origine des gains significativement similaires obtenus et de la même croissance pondérale entre les agneaux des deux lots durant les premières semaines de vie. En effet, une importante mobilisation des réserves a lieu pendant la première phase de la lactation, car les besoins de lactations sont élevés et l'ingestion reste insuffisante. Les lipides, constitués durant les deux derniers tiers de la phase de gestation, constituent la partie essentielle des réserves corporelles mobilisées qui interviennent en fin de gestation pour la croissance fœtale et au démarrage de la lactation. 
L'utilisation aussi bien de l'orge que de la caroube, comme compléments au steaming a contribué, de façon identique, à une constitution de ces réserves jusqu'à la mise bas, d'où un gain, significativement identique, en NEC à la mise bas pour les femelles des deux lots et probablement une augmentation similaire de la production laitière pour les deux lots, ce qui s'est reflété par des poids et des croissances pondérales significativement identiques des agneaux de J10 à J50.

Si les notes d'état corporel finales n'ont pas influencé statistiquement les poids des agneaux et leur croissance pondérale, ces poids (de J10 à J50) seraient corrélés positivement avec le poids à la naissance (J0) (tableau V). Ainsi, les agneaux les plus lourds à la naissance seront ceux qui auront le meilleur poids, du moins jusqu'à J50.

Les deux compléments énergétiques (caroube vs. orge) ayant donné les mêmes performances zootechniques et pondérales, ils peuvent de ce fait se substituer l'un à l'autre.

\section{CONCLUSION}

Les résultats de ce travail montrent qu'un flushing ou un steaming réalisés aussi bien avec un concentré à base d'orge qu'avec de la caroube entière chez des brebis Ouled Djellal assurent des performances de production et de reproduction similaires. En effet, les femelles ayant été complémentées avec les deux compléments énergétiques avant la lutte ont vu leur note d'état corporel s'améliorer de façon similaire, d'où la contribution aussi bien de l'orge que de la caroube à la reconstitution des réserves corporelles des brebis d'autant plus si celle-ci est réalisée au stade propice. En outre, les performances de reproduction ont été statistiquement les mêmes que ça soit pour les femelles du lot témoin ou expérimental.

Il en est de même pour les femelles complémentées avec l'orge ou la caroube avant leur mise bas, chez qui une amélioration de l'état de chair a été enregistrée sans toutefois une différence significative entre les deux lots. Le même effet a été enregistré concernant la croissance pondérale des agneaux issus des deux lots qui ont vu leurs poids vifs s'améliorer sans toutefois de différence significative.

Ces résultats confirment la nécessité de mieux mâ̂triser les apports alimentaires en période de lutte et avant la mise bas par la distribution d'un concentré à base de caroube entière qui peut substituer un concentré classique à base d'orge et dont les résultats ont été intéressants chez ces catégories d'animaux.

La connaissance des variations des réserves à travers la note d'état corporel (NEC) est nécessaire pour les intégrer dans des programmes de complémentation alimentaire des ovins.

Il serait, tout aussi intéressant de mener l'expérience en comparant des concentrés à base de caroube entière et de pulpe de caroube.

\section{BIBLIOGRAPHIE}

Abbas, K.; Chouya, F. et Madani, T. 2002. Facteurs d'amélioration de la reproduction dans les systèmes ovins en zones semi-arides algériennes. Proceedings des 9 èmes Rencontres Recherches autour des Ruminants. 2002 décembre. Paris.

AOAC. Official Methods of Analysis. 1990. Association of Official Analytical Chemists. $15^{\text {th }}$ ed. Washington. D.C. USA.

Avallone, R., Plessi, M.; Baraldi, M. and Monzani, A. 1997. Determination of chemical composition of carob (Ceratonia siliqua): Protein, fat, carbohydrates, and tannins. J Food Comp and Anal, 10: 166-172.

Baumont, R.; Dulphy, J.P.; Sauvant, D.; Meschy, F.; Aufrere, J. et Peyraud, J.L. 2007. Valeur alimentaire des fourrages et des matières premières: Tables et prévision. In: Alimentation des bovins, ovins et caprins. Quæ éditions. INRA. Paris. pp. 149-179.

Biner, B.; Gubbuk, H.; Karhan, M.; Aksu, M. and Pekmezci, M. 2007. Sugar profiles of the pods of cultivated and wild types of carob bean (Ceratonia siliqua L.). Turkey Food Chem, 100: 1453-1455.

Bocquier, F.; Theriez, M.; Prache, S. et Brelurut, A. 1988. Alimentation des ovins. In: Alimentation des bovins, ovins et caprins. INRA Éditions. Paris. pp. 249-281

Boucif, A.; Azzi, N.; Tainturier, D. et Niar, A. 2007. Variations saisonnières des paramètres reproductifs chez les béliers de deux races locales algériennes. Proceedings des 9 èmes Rencontres Recherches autour des Ruminants. Décembre 2007. Paris.

Brown, G.H. 1988. The statistical comparison of reproduction rats for groups of sheep. Aust J Agric Res, 39: 899-905.

Cabée, M. 1959. Le mouton en Algérie. Bulletin Technique des Ingénieurs des Services Agricoles, 142: 51 1-524.

Chellig, R. 1992. Les races ovines Algériennes. Office des Publications Universitaires. Alger.

Dedieu, B.; Cournut, E. et Gibon, A. 1989. Notations d'état corporel et systèmes d'élevage ovin: diagnostic et conseil pour l'alimentation des troupeaux en Cévennes. INRA Prod Anim, 2: 79-88.

Dehimi, M.L.; Dib, Y. and Slimani, A. 2001. Management of Sheep Reproduction by using the Ram Effect in Mashreq-Maghreb Project Newsletter: Sidi Fredj and M'toussa Communities in Algeria 19: 28-30.

Demarquilly, C.; Grenet, E. et Andrieu, J. 1981. Les constituants azotés des fourrages et la prévision de la valeur azotée des fourrages. In: Andrieu, J.; Demarquilly, C.; Wegat-Litre, E. Theix (Eds.). Prévision de la valeur nutritive des aliments des ruminants. INRA publications. Paris. pp. 129-154.

Dirand, A. 2007. L'élevage du mouton. Edition Educagri. Dijon cedex. France.

Dudouet, C. 2003. La production du mouton. $2^{\text {ème }}$ éd. Editions France agricole. Paris. France.

FAOSTAT. 2010. Statistics of Food and Agriculture Organization of the United Nations.

Gaouar, N. 2011 . Étude de la valeur nutritive de la caroube de différentes variétés Algériennes. Mémoire de Magister. Université Abou Bekr Belkaid. Tlemcen. Algérie. 95 pp.

Guessous, F.; El Hilali, A. et Johnson, W.L. 1988. Influence du taux $\mathrm{d}^{\prime}$ incorporation de la pulpe de caroube sur la digestibilité et l'utilisation des rations par des ovins à l'engraissement. Reprod Nutr Dévelop, 28: 93-94.

Molina, M.P.; Sanna, A.; Molle, G.; Branca, A.; Ruda, R. et Casu, S. 1991. Evolution de l'état corporel des brebis Sardes pendant la lactation et relation avec la productivité en conditions d'élevage intensif. In: Purroy A. (Ed.). Options Méditerranéennes. Série Séminaires, 13: 1991 May 14-16. Zaragoza (Spain). pp. 91-96.

Morand-Fehr, P.; Bas, P.; Hervieu, J. et Sauvant, D. 1990. Estimation de la teneur en lipides des chèvres laitières par diverses méthodes informant sur le métabolisme lipidique ou l'état corporel. Reprod Nutr Dev, 30: 255-256.

Nicoll, M. 1981. Sources of variation in the condition scoring of cows. Irish J Agric Res, 20: 27-33.

Paquay, R. 2004. Le comportement alimentaire du mouton. Filière Ovine et Caprine, 9: 1-7. 
Sauvant, D. 1981. Prévision de la valeur énergétique des aliments concentrés et composés pour les ruminants. In: Andrieu, J.; Demarquilly, C. and Wegat-Litre, E. (Eds.). INRA publications. Paris. pp. 237-258. SPSS Inc. 2008. Released. SPSS Statistics for Windows. Version 17.0. Chicago.

Teillet, B.; Colin, M.; Armengol, J. et Prigent, A.Y. 2011 . Effet d'un extrait de graines de caroube partiellement décortiquées sur les performances deviabilité et de croissance chez le lapin. Proceeding des $14^{\text {èmes }}$ journées sur la recherche cunicole, 22-23 novembre 2011. Le Man. France.
Theriez, M. 1984. Influence de l'alimentation sur les performances de reproduction des ovins. Journées Recherches sur les ovins et caprins. INRA-ITOVIC. pp. 294-326.

Teyssier, J.; Lapeyronie, P.; Vincent, M. et Molenat, G. 1995. État corporel pendant la gestation chez la brebis Mérinos d'Arles en système transhumant. Relations avec le poids à la naissance des agneaux et les performances d'allaitement. In: Purroy, A. (Ed.). Options Méditerranéennes. Série Séminaires. $n^{\circ} 27$. Seminar of the Working Group on Body Condition of Sheep and Goats of the FAO/CIHEAM Network on Sheep and Goats. 1994 Mars 24-31. Zaragoza (Spain). pp. 43-51. 
